\title{
A GENERALIZATION OF MATHIEU SUBSPACES TO MODULES OF ASSOCIATIVE ALGEBRAS
}

\author{
WENHUA ZHAO
}

\begin{abstract}
We first propose a generalization of the notion of Mathieu subspaces of associative algebras $\mathcal{A}$, which was introduced recently in $\mathbf{Z 4}$ and $\mathbf{Z 6}$, to $\mathcal{A}$-modules $\mathcal{M}$. The newly introduced notion in a certain sense also generalizes the notion of submodules. Related with this new notion, we also introduce the sets $\sigma(N)$ and $\tau(N)$ of stable elements and quasi-stable elements, respectively, for all $R$-subspaces $N$ of $\mathcal{A}$-modules $\mathcal{M}$, where $R$ is the base ring of $\mathcal{A}$. We then prove some general properties of the sets $\sigma(N)$ and $\tau(N)$. Furthermore, examples from certain modules of the quasi-stable algebras [Z6, matrix algebras over fields and polynomial algebras are also studied.
\end{abstract}

\section{Introduction}

1.1. Background and Motivation. Once and for all, we fix an arbitrary unital commutative ring $R$ and an associative but not necessarily commutative unital $R$-algebra $\mathcal{A}$. Recall that the following notion has been introduced recently in [Z4] and [Z6].

Definition 1.1. Let $J$ be a $R$-submodule or $R$-subspace of $\mathcal{A}$. We say $J$ is a left (resp., right; two-sided) Mathieu subspace of $\mathcal{A}$ if the following property holds: if $a \in \mathcal{A}$ satisfies $a^{m} \in J$ for all $m \geq 1$, then for each $b, c \in \mathcal{A}$, we have $b a^{m} \in J$ (resp., $a^{m} c \in J ; b a^{m} c \in J$ ) for all $m \gg 0$, i.e., there exists $N \geq 1$ such that $b a^{m} \in J$ (resp., $\left.a^{m} c \in J ; b a^{m} c \in J\right)$ for all $m \geq N$.

Two-sided Mathieu subspaces will also simply be called Mathieu subspaces. A $R$-subspace $M$ of $\mathcal{A}$ is said to be a pre-two-sided Mathieu subspace of $\mathcal{A}$ if it is both left and right Mathieu subspace of $\mathcal{A}$. Note that the pre-two-sided Mathieu subspaces were previously called twosided Mathieu subspace or Mathieu subspaces in [Z4].

2000 Mathematics Subject Classification. 16D10, 16D99.

Key words and phrases. Mathieu subspaces of associative algebras, Mathieu subspaces of modules of associative algebras, (quasi-)stable elements, (quasi-)stable algebras, (quasi-)stable modules.

The author has been partially supported by NSA Grant H98230-10-1-0168. 
The introduction of the notion of Mathieu subspaces in [Z4] and [Z6] was mainly motivated by the studies of the Jacobian conjecture [K] (see also BCW and [E1]), the Dixmier conjecture [D] (see also [T], BK and $[\mathrm{AE}]$ ), the Mathieu conjecture [Ma], the vanishing conjecture [Z1], [Z2], [Z5], [EWiZ] and more recently, the image conjecture [Z3], as well as many other related open problems. Actually, both the Mathieu conjecture and the image conjecture imply the Jacobian conjecture (and hence also the Dixmier conjecture), and both are (open) problems on whether or not certain subspaces of some algebras are Mathieu subspaces. For some recent developments on Mathieu subspaces, see [Z6], [FPYZ], [EWrZ1], EWrZ2], WZ] and [EZ]. For a recent survey on the the image conjecture and it's connections with some other problems, see [E2].

Note that every left ideal of $\mathcal{A}$ is a left Mathieu subspace of $\mathcal{A}$. This is also the case for right or two-sided ideals. Therefore, the notion of Mathieu subspaces can be viewed as a natural generalization of the notion of ideals.

On the other hand, the notion of ideals of associative algebras $\mathcal{A}$ has a natural generalization to their modules. Namely, viewing $\mathcal{A}$ itself as a left (resp., right or two-sided) $\mathcal{A}$-module in the canonical way, every left (resp., right or two-sided) ideal of $\mathcal{A}$ is a submodule of the left (resp., right or two-sided) $\mathcal{A}$-module $\mathcal{A}$.

Naturally, one may wonder whether or not there is also a generalization of Mathieu subspaces of associative algebras to their modules, which is parallel to the above generalization of the ideals to submodules, such that the following diagram of notions commutes:

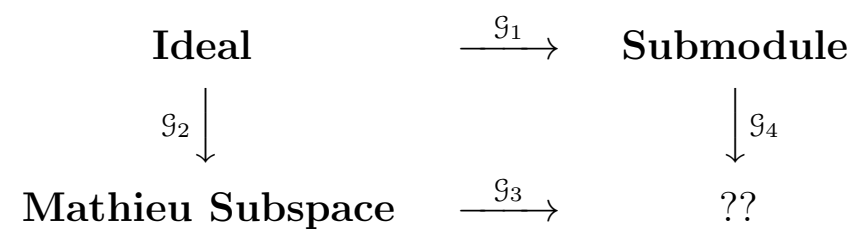

where the "maps" $\mathcal{G}_{i}(1 \leq i \leq 4)$ in the diagram denote the generalizations of the corresponding notions.

Note that the generalization from the notion of ideals to the notion of submodules is crucial in the theories of various algebras (associative algebras, Lie algebras, etc.). We believe that the generalization $\mathcal{G}_{3}$ in the diagram above is also important, not only for the study of modules of associative algebras but also for the study of Mathieu subspaces of associative algebras.

In this paper, we first complete the commutative diagram above by introducing the notion of what we call Mathieu subspaces for modules 
$\mathcal{M}$ of associative algebras $\mathcal{A}$ (see Definition 1.2 below). Related with this new notion, we also introduce the sets $\sigma(N)$ and $\tau(N)$ of stable elements and quasi-stable elements, respectively, for all $R$-subspaces $N$ of $\mathcal{A}$-modules $\mathcal{M}$ (see the discussions in Subsection 1.2 below and also Definition 3.1 in Section 3). We then study some general properties of the sets $\sigma(N)$ and $\tau(N)$. Furthermore, examples from certain modules of the quasi-stable algebras [Z6] (see also Definition 2.7), matrix algebras over fields and polynomial algebras are also studied.

1.2. To Complete the Commutative Diagram. To be more precise, let's first recall the following terminologies and conventions introduced in [Z6], which will also be used throughout this paper.

Note first that for Mathieu subspaces and ideals, as well as for the new notions to be defined below, we have several different cases: left, right and (pre-)two-sided. Very often, it is necessary and important to treat all these cases. For simplicity, following [Z6] we introduce the short terminology $\vartheta$-Mathieu subspaces for Mathieu subspaces, where $\vartheta$ stands for left, right, pre-two-sided, or two-sided. Similarly, we introduce the terminology $\vartheta$-ideals for ideals, except for the specification $\vartheta=$ "pre-two-sided", we also set $\vartheta$-ideals to mean two-sided ideals.

In other words, the reader should read the letter $\vartheta$ as an index or a variable with four possible choices or "values". However, to avoid repeating the phrase "for every specification of $\vartheta$ " or "for every $\vartheta$ " infinitely many times, we will simply leave $\vartheta$ unspecified for the statements or propositions which hold for all the four specifications of $\vartheta$. For example, by saying "Any $\vartheta$-ideal of $\mathcal{A}$ is a $\vartheta$-Mathieu subspace of $\mathcal{A}$ " or " $\mathcal{A}$ has at least one proper $\vartheta$-Mathieu subspace", we mean that the statement holds for every fixed specification of $\vartheta$ : left, right, pre-two-sided or two-sided.

Furthermore, in the case that only the two-sided case is concerned, the variable $\vartheta$ will be simply dropped, e.g., the words ideals and Mathieu subspaces (without the variable $\vartheta$ ) always mean the two-sided ideals and two-sided Mathieu subspaces, respectively.

Now, let $R$ and $\mathcal{A}$ be as before and $\mathcal{M}$ a left $\mathcal{A}$-module. For any $u \in \mathcal{M}$ and any subset $N$ of $\mathcal{M}$, we set

$$
(N: u):=\{a \in \mathcal{A} \mid a u \in N\} .
$$

With the terminologies and notations fixed above, we can now introduce the new notion claimed in the previous subsection as follows.

Definition 1.2. Let $u \in \mathcal{M}$ and $N$ be a $R$-subspace or $R$-submodule of $\mathcal{M}$. We say that $N$ is a $\vartheta$-Mathieu subspace of $\mathcal{M}$ with respect to $u$ if $(N: u)$ is a $\vartheta$-Mathieu subspace of $\mathcal{A}$. 
In a similar way as above we can define $\vartheta$-Mathieu subspaces for right $\mathcal{A}$-modules as well. Since every right $\mathcal{A}$-module is a left $\mathcal{A}^{o p}$-module, where $\mathcal{A}^{\text {op }}$ denotes the opposite algebra of $\mathcal{A}$, we may (and also will) focus only on the $\vartheta$-Mathieu subspaces of left $\mathcal{A}$-modules. Therefore, throughout this paper, all $\mathcal{A}$-modules will be assumed to be left $\mathcal{A}$ modules unless stated otherwise. In particular, when we view $\mathcal{A}$ itself as an $\mathcal{A}$-module, we always mean the left $\mathcal{A}$-module over the $R$-space $\mathcal{A}$ with the left action given by the algebra product of $\mathcal{A}$.

The main ideas behind Definition 1.2 are as follows.

Note first that in general $\mathcal{A}$-modules $\mathcal{M}$ have no product operations. Therefore, we cannot generalize directly the notion of $\vartheta$-Mathieu subspaces of the algebra $\mathcal{A}$ to $\mathcal{M}$, as we did for the generalization of left ideals of $\mathcal{A}$ to submodules of $\mathcal{M}$.

But, on the other hand, for every $R$-subspace $J$ of the $\mathcal{A}$-module $\mathcal{A}$, we have $\left(J: 1_{\mathcal{A}}\right)=J$. Hence, $J$ is a $\vartheta$-Mathieu subspace of the algebra $\mathcal{A}$ iff $J$ is a $\vartheta$-Mathieu subspace of the $\mathcal{A}$-module $\mathcal{A}$ with respect to $1_{\mathcal{A}} \in$ $\mathcal{A}$. Therefore, from this point of view we see that the notion of Mathieu subspaces of $\mathcal{A}$-modules given in Definition 1.2 indeed generalizes the notion of Mathieu subspaces of the algebra $\mathcal{A}$. Actually, as we will show later in Section 3 (see Corollary 3.14 and Remark 3.15), with $\vartheta=$ "left" it also generalizes the notion of submodules of $\mathcal{A}$-modules $\mathcal{M}$ in the sense that: for every $R$-subspace $N$ of $\mathcal{M}, N$ is a submodule of $\mathcal{M}$ iff $N$ is a left Mathieu subspace of $\mathcal{M}$ with respect to all elements $u \in \mathcal{M}$.

In contrast to ideals of $\mathcal{A}$ or submodules of $\mathcal{A}$-modules $\mathcal{M}$, the $\vartheta$ Mathieu subspaces $N$ of $\mathcal{A}$-modules $\mathcal{M}$ defined in Definition 1.2 depend on some referring elements $u \in \mathcal{M}$. So it is important and also convenient to consider the set of all the referring elements of $N$, i.e., the set of all the elements $u \in \mathcal{M}$ with respect to which $N$ is a $\vartheta$-Mathieu subspace of $\mathcal{M}$. We denote this set by $\tau_{\vartheta}(N)$ and call it the set of $\vartheta$-quasi-stable elements of the $R$-subspace $N \subseteq \mathcal{M}$. Similarly, we let $\sigma_{\vartheta}(N)$ denote the set of all the elements $u \in \mathcal{M}$ such that $(N: u)$ is a $\vartheta$-ideal of $\mathcal{A}$, and call it the set of $\vartheta$-stable elements of $N$. For the justifications of the terminologies of $\vartheta$-quasi-stableness and $\vartheta$-stableness, see Definition 3.1 and also the followed discussions.

Even though our main concerns in this paper are on the sets $\tau_{\vartheta}(N)$ of $\vartheta$-quasi-stable elements of $R$-subspaces $N$ of $\mathcal{A}$-modules $\mathcal{M}$, for the purpose of comparison we also give all the parallel results (to that of $\left.\tau_{\vartheta}(N)\right)$ for the set $\sigma_{\vartheta}(N)$ of $\vartheta$-stable elements of $N$. But, since the proofs for the case of $\sigma_{\vartheta}(N)$ are very often similar to (and actually simpler than) the proofs for the case of $\tau_{\vartheta}(N)$, we will leave to the reader most of the proofs for the case of $\sigma_{\vartheta}(N)$. 
1.3. Arrangement. In Section 2, we recall some results on $\vartheta$-Mathieu subspaces of associative algebras $\mathcal{A}$, which will be needed later in this paper. Some of these results will also be generalized in Section 3 to $\vartheta$-Mathieu subspaces of $\mathcal{A}$-modules.

In Section 3, we study some general properties of the sets $\tau_{\vartheta}(N)$ and $\sigma_{\vartheta}(N)$ of $\vartheta$-quasi-stable elements and $\vartheta$-stable elements, respectively, of $R$-subspaces $N$ of $\mathcal{A}$-modules $\mathcal{M}$.

Note that the processes of taking the sets $\tau_{\vartheta}(N)$ and $\sigma_{\vartheta}(N)$ of $R$ subspaces $N$ of $\mathcal{M}$ can be viewed as two maps from the set of $R$ subspaces $N$ of $\mathcal{M}$ to the set of subsets of $\mathcal{M}$. In this section, we mainly study the relations of these two maps with some other operations on $R$-subspaces of $\mathcal{M}$, such as the relation with the operation of finite intersections in Proposition 3.7, the relation with the pulling-back by the homomorphisms of $\mathcal{A}$-modules in Proposition 3.16, and the relation with the pulling-back by homomorphisms of algebras in Proposition 3.22 , etc. Some consequences of the propositions above are also derived.

Another main result of this section is Theorem 3.11, which claims that for every $R$-subspace $N$ of $\mathcal{M}$, the intersections of both $\tau_{\vartheta}(N)$ and $\sigma_{\vartheta}(N)$ with $N$ itself are the same as the (unique) $\mathcal{A}$-submodule of $\mathcal{M}$, denoted by $I_{N}$, which is maximum among all the $\mathcal{A}$-submodules of $\mathcal{M}$ contained in $N$. For short, in this paper we call the $\mathcal{A}$-submodule $I_{N}$ the maximum $\mathcal{A}$-submodule of $N$. Some consequences of this theorem are also derived in Corollaries 3.12 3.14.

In Section 4, we first introduce in Definition 4.1 the notions of the $\vartheta$-quasi-stable and $\vartheta$-stable $\mathcal{A}$-modules. The relations of these two new notions with the notions of $\vartheta$-quasi-stable and $\vartheta$-stable $R$-algebras introduced in [Z6] (see also Definition 2.7) are given in Proposition 4.4. More explicitly, a $R$-algebra $\mathcal{A}$ is $\vartheta$-quasi-stable (resp., $\vartheta$-stable) iff all its modules are $\vartheta$-quasi-stable (resp., $\vartheta$-stable).

One special property of $\vartheta$-quasi-stable (resp., $\vartheta$-stable) modules $\mathcal{M}$ is that for all $R$-subspaces $N$ of $\mathcal{M}$, by Theorem 3.11 we have $\tau_{\vartheta}(N)=$ $I_{N} \cup N^{c}$ (resp., $\sigma_{\vartheta}(N)=\tau_{\vartheta}(N)=I_{N} \cup N^{c}$ ), where $I_{N}$ is the maximum $\mathcal{A}$-submodule of $N$ (defined above) and $N^{c}$ denotes the complement of $N$ in $\mathcal{M}$.

On the other hand, it has been shown in [Z6] (see also Proposition 2.8) that for every $R$-algebra $\mathcal{A}$, if $\mathcal{A}$ is integral over $R$ and every element of $\mathcal{A}$ is either invertible or nilpotent, then $\mathcal{A}$ is quasi-stable. Consequently, every left or right Artinian $R$-algebra $\mathcal{A}$, which is integral over $R$, is quasi-stable. The $\vartheta$-quasi-stable and $\vartheta$-stable algebras over fields have been classified in [Z6] (see also Theorem 2.10 and Propositions 2.11 and 2.12). 
Therefore, we get a family of associative algebras $\mathcal{A}$ such that for all $\mathcal{A}$-modules $\mathcal{M}$, the sets $\tau_{\vartheta}(N)$ for all $R$-subspaces $N$ of $\mathcal{M}$ can be determined explicitly (see Corollaries 4.5 and 4.6).

In Section 5, we study the cases for two modules of the matrix algebras $M_{n}(K)(n \geq 1)$ over an arbitrary field $K$. First, we determine in Proposition 5.1 the sets $\tau_{\vartheta}(N)$ and $\sigma_{\vartheta}(N)$ explicitly for all $K$-subspaces $N$ of the $M_{n}(K)$-module $K^{n}$ (with the canonical left action). Second, we view $M_{n}(K)$ itself as a (left) $M_{n}(K)$-module in the canonical way and determine in Proposition 5.3 the sets $\tau_{\vartheta}(N)$ and $\sigma_{\vartheta}(N)$ explicitly for all co-dimension one $K$-subspaces $N$ of $M_{n}(K)$.

In Section [6, motivated by Conjecture 3.2 in [Z4] on integrals of polynomials, we study the cases for two modules of the polynomial algebras over fields $K$. We first consider a family of $K$-subspaces $N_{B, \alpha}$ defined in Eq. (6.2) of the polynomial algebra in several variables over an arbitrary field $K$, and derive the sets $\sigma\left(N_{B, \alpha}\right)$ and $\tau\left(N_{B, \alpha}\right)$ explicitly in Proposition 6.3. We then consider a family of $\mathbb{C}$-subspaces $N_{q}$ defined in Eq. (6.8) of the polynomial algebra in one variable over the complex field $\mathbb{C}$, and derive the sets $\sigma\left(N_{q}\right)$ and $\tau\left(N_{q}\right)$ explicitly in Proposition 6.7 .

\section{Some Properties of $\vartheta$-Mathieu Subspaces of Associative Algebras}

Let $R$ and $\mathcal{A}$ be as fixed in the previous section. We denote by $1_{\mathcal{A}}$ or simply 1 the identity element of the $R$-algebra $\mathcal{A}$. The sets of units or invertible elements of $R$ and $\mathcal{A}$ will be denoted by $R^{\times}$and $\mathcal{A}^{\times}$, respectively. All the notations, conventions and terminologies fixed in the previous section will also be in force throughout the paper.

Let $J$ be an arbitrary subset of $\mathcal{A}$. Following [Z6] we define the radical of $J$, denoted by $\sqrt{J}$, to be $\sqrt{J}:=\left\{a \in \mathcal{A} \mid a^{m} \in J\right.$ when $\left.m \gg 0\right\}$. Note that $\sqrt{\mathcal{A}}=\mathcal{A}$, and $\sqrt{0}$ is the set of all nilpotent elements of $\mathcal{A}$. We will also denote $\sqrt{0}$ by nil $(\mathcal{A})$ since when $\mathcal{A}$ is commutative, $\sqrt{0}$ is the same as the nilradical of $\mathcal{A}$.

For any $a \in \mathcal{A}$ and $\vartheta \neq$ "pre-two-sided", we denote by $(a)_{\vartheta}$ the $\vartheta$ ideal generated by $a$. For the case $\vartheta=$ "pre-two-sided", we set $(a)_{\vartheta}:=$ $A a+a A$. Also, as fixed in the previous section, when $\vartheta=$ "two-sided", we will simply drop the variable $\vartheta$ from the notation above, i.e., $(a)$ denotes the (two-sided) ideal of $\mathcal{A}$ generated by the element $a$.

Furthermore, for each $a \in \mathcal{A}$, we also set

$$
a^{-1} J:=\{b \in \mathcal{A} \mid a b \in J\} .
$$


Note that $a^{-1} J$ is an abusing notation since $a$ might not be invertible in $\mathcal{A}$.

In this section, we mainly recall some properties of $\vartheta$-Mathieu subspaces of $R$-algebras $\mathcal{A}$, which will be needed later in this paper.

Let's start with the following lemma, which is very simple but provides a family of (two-sided) Mathieu subspaces.

Lemma 2.1. Let $J$ be a $R$-subspace of $\mathcal{A}$ such that $\sqrt{J} \subseteq \operatorname{nil}(\mathcal{A})$. Then every $R$-subspace $H$ of $J$ is a Mathieu subspace of $\mathcal{A}$.

The next lemma proved in [Z6] provides a different point of view to see that the notion of $\vartheta$-Mathieu subspaces is indeed a natural generalization of that of $\vartheta$-ideals.

Lemma 2.2. Let $J$ be a $R$-subspace of a $R$-algebra $\mathcal{A}$. Then the following statements hold.

i) $J$ is a left ideal of $\mathcal{A}$ iff for any $a \in \mathcal{A}$, we have

$$
J \subseteq a^{-1} J .
$$

ii) $J$ is a left Mathieu subspace of $\mathcal{A}$ iff for any $a \in \mathcal{A}$, we have

$$
\sqrt{J} \subseteq \sqrt{a^{-1} J}
$$

iii) $J$ is a right ideal of $\mathcal{A}$ iff for any $a \in \mathcal{A}$, we have

$$
J \subseteq(J: a) .
$$

iv) $J$ is a right Mathieu subspace of $\mathcal{A}$ iff for any $a \in \mathcal{A}$, we have

$$
\sqrt{J} \subseteq \sqrt{(J: a)}
$$

v) $J$ is a (two-sided) ideal of $\mathcal{A}$ iff for any $a, b \in \mathcal{A}$, we have

$$
J \subseteq\left(a^{-1} J: b\right) .
$$

vi) $J$ is a (two-sided) Mathieu subspace of $\mathcal{A}$ iff for any $a, b \in \mathcal{A}$, we have

$$
\sqrt{J} \subseteq \sqrt{\left(a^{-1} J: b\right)}
$$

The following two simple lemmas, first noticed in [Z4], will be very useful for our later arguments.

Lemma 2.3. Any proper $R$-subspace $J$ of $\mathcal{A}$ with $1_{\mathcal{A}} \in J$ is not a $\vartheta$-Mathieu subspace of $\mathcal{A}$.

Lemma 2.4. For every finite family of $\vartheta$-Mathieu subspaces $J_{i}(1 \leq$ $i \leq k)$ of $\mathcal{A}$, the intersection $\bigcap_{i=1}^{k} J_{i}$ is also a $\vartheta$-Mathieu subspace of $\mathcal{A}$. 
Note that in contrast to the case of $\vartheta$-ideals, intersections of infinitely many $\vartheta$-Mathieu subspaces of $\mathcal{A}$ are not always $\vartheta$-Mathieu subspaces of $\mathcal{A}$, e.g., see Example 4.17 in [Z6].

The following two propositions will also be important for our later arguments.

Proposition 2.5. ([Z6]) Let $I$ be an ideal of $\mathcal{A}$ and $J$ a $R$-subspace of $\mathcal{A}$ which contains $I$. Then $J$ is a $\vartheta$-Mathieu subspace of $\mathcal{A}$ iff $J / I$ is a $\vartheta$-Mathieu subspace of $\mathcal{A} / I$.

Proposition 2.6. ([Z4]) Let $\phi: \mathcal{A} \rightarrow \mathcal{B}$ be a homomorphism of $R$ algebras. Then for every $\vartheta$-Mathieu subspace $N$ of $\mathcal{B}, \phi^{-1}(N)$ is a $\vartheta$-Mathieu subspace of $\mathcal{A}$.

Next, we recall the following notions introduced in [Z6].

Definition 2.7. A $R$-algebra $\mathcal{A}$ is said to be $\vartheta$-quasi-stable (resp., $\vartheta$ stable) if every $R$-subspace $J$ of $\mathcal{A}$ with $1 \notin J$ is a $\vartheta$-Mathieu subspace (resp., $\vartheta$-ideal) of $\mathcal{A}$.

A family of quasi-stable $R$-algebras is given by the following proposition which was proved in Proposition 7.4 and Corollary 7.5 in [Z6].

Proposition 2.8. Let $\mathcal{A}$ be a $R$-algebra such that $\mathcal{A}$ is integral over $R$ and every element of $\mathcal{A}$ is either invertible or nilpotent. Then $\mathcal{A}$ is a quasi-stable $R$-algebra.

Consequently, every left or right Artinian local $R$-algebra, which is integral over $R$, is quasi-stable.

Now, we assume that the base $\operatorname{ring} R$ is a field $K$ and $\mathcal{A}$ is a $K$ algebra. A subset $S \subseteq \mathcal{A}$ is said to be algebraic over $K$ if every element $a \in S$ is algebraic over $K$, i.e., a satisfies a nonzero polynomial in one variable with coefficients in $K$. Recall also that an element $a \in \mathcal{A}$ is said to be an idempotent if $a^{2}=a$.

With the terminologies fixed above, we have the following characterization proved in [Z6] for the $\vartheta$-Mathieu subspaces $J$ of $K$-algebras $\mathcal{A}$, whose radical $\sqrt{J}$ is algebraic over $K$.

Theorem 2.9. Let $J$ be a $K$-subspace of a $K$-algebra $\mathcal{A}$ such that $\sqrt{J}$ is algebraic over $K$. Then $J$ is a $\vartheta$-Mathieu subspace of $\mathcal{A}$ iff for every idempotent $e \in J$, we have $(e)_{\vartheta} \subseteq J$.

Furthermore, the $\vartheta$-quasi-stable algebras over fields $K$ defined above in Definition 2.7 have been classified in [Z6] as follows. 
Theorem 2.10. Let $K$ be an arbitrary field and $\mathcal{A}$ a $K$-algebra. Then $\mathcal{A}$ is $\vartheta$-quasi-stable iff either $\mathcal{A} \simeq K \dot{+} K$, or $\mathcal{A}$ is an algebraic local $K$ algebra, where $K \dot{+} K$ is the $K$-algebra with $K \times K$ as the base $K$-vector space and the component-wise product as the algebra product.

One remark on the theorem above is that for algebras over fields, the $\vartheta$-quasi-stableness actually does not depend on the specialization of $\vartheta$. More precisely, an algebra $\mathcal{A}$ over a field $K$ is one-sided (left or right) quasi-stable iff it is (two-sided) quasi-stable. By Proposition 2.12 below, this is also the case for the $\vartheta$-stableness.

Proposition 2.11. For any algebraic $K$-algebra $\mathcal{A}$, the following statements are equivalent:

1) $\mathcal{A}$ is local;

2) $\mathcal{A}$ has no idempotents except $0,1 \in \mathcal{A}$;

3) each element of $\mathcal{A}$ is either nilpotent or invertible.

Finally, let's point out that the $\vartheta$-stable $K$-algebras have also been classified in [Z6] as follows.

Proposition 2.12. Let $K$ be an arbitrary field and $\mathcal{A}$ a $K$-algebra. Then $\mathcal{A}$ is $\vartheta$-stable iff one of the following two statements holds:

1) $\mathcal{A}=K$;

2) $K \simeq \mathbb{Z}_{2}$ and $\mathcal{A} \simeq \mathbb{Z}_{2}+\mathbb{Z}_{2}$.

\section{Mathieu Subspaces of Modules of Associative Algebras}

Let $R$ and $\mathcal{A}$ be as before and $\mathcal{M}$ an arbitrary (left) $\mathcal{A}$-module. Recall that we have set in Section 1.2 the convention that all $\mathcal{A}$-modules in this paper will be left $\mathcal{A}$-modules unless stated otherwise. In particular, when we say $\mathcal{A}$ itself is an $\mathcal{A}$-module, we always mean that the left $\mathcal{A}$ module on the $R$-space $\mathcal{A}$ with the action given by the algebra product of $\mathcal{A}$ (from left).

Recall also that we have defined in Definition 1.2 the $\vartheta$-Mathieu subspaces for the $\mathcal{A}$-module $\mathcal{M}$. But, for convenience we also fix the following terminologies.

Definition 3.1. With the notations and the setting as above, let $u \in \mathcal{M}$ and $N$ a $R$-subspace of $\mathcal{M}$. We say that

1) $u$ is $\vartheta$-stable with respect to $N$ if $(N: u)$ is a $\vartheta$-ideal of $\mathcal{A}$;

2) $u$ is $\vartheta$-quasi-stable with respect to $N$ if $(N: u)$ is a $\vartheta$-Mathieu subspace of $\mathcal{A}$.

By the definition above, the sets $\tau_{\vartheta}(N)$ and $\sigma_{\vartheta}(N)$ defined on page 4 in Section 1.2 are respectively the sets of $\vartheta$-quasi-stable elements and $\vartheta$-stable elements of the $R$-subspace $N$ of $\mathcal{M}$. 
The terminologies in Definition 3.1 can be justified as follows for the case $\vartheta=$ "left".

If $u$ is left stable with respect to $N$ and some $a \in \mathcal{A}$ maps $u$ into the $R$-subspace $N$ of $\mathcal{M}$, i.e., au $\in N$, then no element $b \in \mathcal{A}$ can map au outside of $N$. In other words, au will stay inside $N$ "forever" under the action of $\mathcal{A}$.

If $u$ is left quasi-stable with respect to $N$ (equivalently, $N$ is a left Mathieu subspace of $\mathcal{M}$ with respect to $u$ ) and for some $a \in \mathcal{A}$, all the terms of the sequence $\left\{a^{m} u\right\}$ lie inside $N$, then for any $b \in \mathcal{A}$, all but finitely many terms of the sequence $\left\{b\left(a^{m} u\right)\right\}$ also lie inside $N$. In other words, no element $b \in \mathcal{A}$ can map infinitely many terms of the sequence $\left\{a^{m} u\right\}$ outside the $R$-subspace $N$.

The terminologies of the right stableness and right quasi-stableness can be interpreted parallelly but with slightly different meanings.

From Definitions 1.2 and 3.1 , it is easy to see that for every $R$ subspace $J$ of $\mathcal{A}$, we have that $J$ is a $\vartheta$-Mathieu subspace (resp., $\vartheta$ ideal) of the $R$-algebra $\mathcal{A}$, iff $J$ as a $R$-subspace of the $\mathcal{A}$-module $\mathcal{A}$ is $\vartheta$-quasi-stable (resp., $\vartheta$-stable) with respect to the identity element $1_{\mathcal{A}} \in \mathcal{A}$, iff $1_{\mathcal{A}} \in \tau_{\vartheta}(N)$ (resp., $1_{\mathcal{A}} \in \sigma_{\vartheta}(N)$ ).

In this section, we mainly study some general properties of the sets $\tau_{\vartheta}(N)$ and $\sigma_{\vartheta}(N)$ of $\vartheta$-quasi-stable elements and $\vartheta$-stable elements, respectively, of $R$-subspaces $N$ of $\mathcal{A}$-modules $\mathcal{M}$. For a summary of the main results in this section, see the arrangement description for this section in Subsection 1.3.

We start with the following two simple lemmas whose proofs are very straightforward and will be skipped here.

Lemma 3.2. For any $\mathcal{A}$-module $\mathcal{M}$, we have

$$
\sigma_{\vartheta}(\mathcal{M})=\tau_{\vartheta}(\mathcal{M})=\mathcal{M} \text {. }
$$

Furthermore, when $\vartheta=$ "left", we have

$$
\sigma_{\vartheta}(0)=\tau_{\vartheta}(0)=\mathcal{M} .
$$

Note that when $\vartheta \neq$ "left", Eq. (3.2) does not necessarily hold, e.g., see Proposition [5.1, ii) in later Section 5.

Lemma 3.3. For each $R$-subspace $N$ of the $\mathcal{A}$-module $\mathcal{M}$, we have,

i) $\sigma_{\vartheta}(N) \subseteq \tau_{\vartheta}(N)$.

ii) $0 \in \sigma_{\vartheta}(N)$ and hence, $0 \in \tau_{\vartheta}(N)$.

iii) $\sigma_{\vartheta}(N)$ and $\tau_{\vartheta}(N)$ are closed under the actions of the elements of $R^{\times} \cup\{0\}$. In particular, when the base ring $R$ is a field, $\sigma_{\vartheta}(N)$ and $\tau_{\vartheta}(N)$ are $R$-cones. 
Actually, when $\vartheta=$ "left", $\sigma_{\vartheta}(N)$ is always closed under the action of $R$ even in the case that $R$ is not a field. More precisely, the following lemma also holds.

Lemma 3.4. Let $\vartheta=$ "left" and $a \in \mathcal{A}$. Then for each $R$-subspace $N \subseteq \mathcal{M}$, we have $a \sigma_{\vartheta}(N) \subseteq \sigma_{\vartheta}(N)$.

Proof: Let $u \in \sigma_{\vartheta}(N)$ (with $\vartheta=$ "left"). Then $(N: u)$ is a left ideal of $\mathcal{A}$. Note that in general the following equation always holds:

$$
(N: a u)=((N: u): a) .
$$

Furthermore, it is also well-known and easy to check that for every left ideal $J$ of $\mathcal{A}$ and $b \in \mathcal{A},(J: b)$ is also a left ideal of $\mathcal{A}$. Then it follows from this fact and Eq. (3.3) that $(N: a u)$ is also a left ideal of $\mathcal{A}$. Therefore, we have $a u \in \sigma_{\vartheta}(N)$, whence the lemma follows.

One remark on Lemma 3.3, iii) and Lemma 3.4 is that $\sigma_{\vartheta}(N)$ and $\tau_{\vartheta}(N)$ in general are not closed under the addition of $\mathcal{A}$ (see the examples to be discussed in later Sections 46).

Next, we give some characterizations for the $\vartheta$-stable elements and $\vartheta$-quasi-stable elements of $R$-subspaces of $\mathcal{A}$-modules, from which we also get some characterizations for $\vartheta$-Mathieu subspaces of $\mathcal{A}$-modules.

Lemma 3.5. Let $u \in \mathcal{M}$ and $N$ a $R$-subspace of $\mathcal{M}$. Set

$$
a^{-1} N:=\{v \in \mathcal{M} \mid a v \in N\} .
$$

Then the following statements holds:

i) $u$ is left stable with respect to $N$ iff for every $a \in \mathcal{A}$, we have

$$
(N: u) \subseteq\left(a^{-1} N: u\right),
$$

ii) $u$ is left quasi-stable with respect to $N$ iff for every $a \in \mathcal{A}$, we have

$$
\sqrt{(N: u)} \subseteq \sqrt{\left(a^{-1} N: u\right)}
$$

iii) $u$ is right stable with respect to $N$ iff for every $a \in \mathcal{A}$, we have

$$
(N: u) \subseteq(N: a u) .
$$

iv) $u$ is right quasi-stable with respect to $N$ iff for every $a \in \mathcal{A}$, we have

$$
\sqrt{(N: u)} \subseteq \sqrt{(N: a u)}
$$

v) $u$ is (two-sided) stable with respect to $N$ iff for every $a, b \in \mathcal{A}$, we have

$$
(N: u) \subseteq\left(a^{-1} N: b u\right) .
$$


vi) $u$ is (two-sided) quasi-stable with respect to $N$ iff for every $a, b \in$ $\mathcal{A}$, we have

$$
\sqrt{(N: u)} \subseteq \sqrt{\left(a^{-1} N: b u\right)} .
$$

Proof: $\quad$ Note first that for any $a \in \mathcal{A}$ and $u \in \mathcal{M}$, by Eqs. (1.2), (2.1) and (3.4) we have the following equation:

$$
\left(a^{-1} N: u\right)=a^{-1}(N: u) .
$$

Then by the equation above and Eq. (3.3), it is easy to see that the lemma follows immediately from Lemma 2.2 and Definition 3.1.

The next lemma says that the operations of taking $\sigma_{\vartheta}(N)$ and $\tau_{\vartheta}(N)$ of $R$-subspaces $N$ of the $\mathcal{A}$-module $\mathcal{M}$ commute with the operation of taking the set $(S: u)$ of subsets $S \subseteq \mathcal{M}$ with respect to elements $u \in \mathcal{M}$.

Lemma 3.6. Let $u \in \mathcal{M}$ and $N$ be a $R$-subspace of $\mathcal{M}$. Then we have

$$
\begin{aligned}
& \left(\sigma_{\vartheta}(N): u\right)=\sigma_{\vartheta}(N: u), \\
& \left(\tau_{\vartheta}(N): u\right)=\tau_{\vartheta}(N: u) .
\end{aligned}
$$

Proof: For each $a \in \mathcal{A}$, we have that $a \in\left(\tau_{\vartheta}(N): u\right)$, iff $a u \in \tau_{\vartheta}(N)$, iff $(N: a u)$ is a $\vartheta$-Mathieu subspace of $\mathcal{A}$, iff $((N: u): a)$ by Eq. (3.3) is a $\vartheta$-Mathieu subspace of $\mathcal{A}$, iff $a \in \tau_{\vartheta}(N: u)$. Hence, Eq. (3.7) follows. Eq. (3.6) can be proved similarly.

The relations of the operations of taking $\sigma_{\vartheta}(N)$ and $\tau_{\vartheta}(N)$ on $R$ subspaces $N$ of $\mathcal{M}$ with the operation of intersection is given by the following proposition.

Proposition 3.7. Let $N_{i}(i \in I)$ be any collection of $R$-subspaces of $\mathcal{M}$. Then the following statements hold:

$$
\bigcap_{i \in I} \sigma_{\vartheta}\left(N_{i}\right) \subseteq \sigma_{\vartheta}\left(\bigcap_{i \in I} N_{i}\right) .
$$

ii) if the cardinal number $|I|<\infty$, then we have

$$
\bigcap_{i \in I} \tau_{\vartheta}\left(N_{i}\right) \subseteq \tau_{\vartheta}\left(\bigcap_{i \in I} N_{i}\right) .
$$

Proof: Note first that for each $u \in \mathcal{M}$, by Eq. (1.2) we have

$$
\left(\left(\bigcap_{i \in I} N_{i}\right): u\right)=\bigcap_{i \in I}\left(N_{i}: u\right) \text {. }
$$


Then it is easy to see that Eq. (3.8) follows from the equation above and the fact that the intersection of every collection of $\vartheta$-ideals of $\mathcal{A}$ is also a $\vartheta$-ideal of $\mathcal{A}$, and Eq. (3.9) follows from the equation above and Lemma 2.4.

Corollary 3.8. Let $u \in \mathcal{M}$ and $N_{i}(1 \leq i \leq k) \vartheta$-Mathieu subspaces of $\mathcal{M}$ with respect to $u$. Then $\bigcap_{i=1}^{k} N_{i}$ is also a $\vartheta$-Mathieu subspace of $\mathcal{M}$ with respect to $u$.

Remark 3.9. Note that submodules and $\vartheta$-Mathieu subspaces are closed under the operation of finite intersection (see Lemma 2.4). By the corollary above, we see that this property is preserved under the generalizations $\mathcal{G}_{i}(i=3,4)$ in the diagram (1.1) from the notions of submodules and $\vartheta$-Mathieu subspaces, respectively, to the notion of $\vartheta$-Mathieu subspaces of $\mathcal{A}$-modules $\mathcal{M}$.

Next, for every $R$-subspace $N$ of $\mathcal{M}$, we study the intersections of $\tau_{\vartheta}(N)$ and $\sigma_{\vartheta}(N)$ with $N$ itself. In contrast to the sets $\tau_{\vartheta}(N)$ and $\sigma_{\vartheta}(N)$ themselves, their intersections with $N$ are always $\mathcal{A}$-submodules (see Theorem 3.11 below). Let's first prove the following lemma.

Lemma 3.10. Let $u \in \mathcal{M}$ and $N$ be a $R$-subspace of $\mathcal{M}$. Then we have i) $u \in N \cap \sigma_{\vartheta}(N)$ iff $\mathcal{A} u \subseteq N$.

ii) $u \in N \cap \tau_{\vartheta}(N)$ iff $\mathcal{A} u \subseteq N$.

Proof: Again, here we just give a proof for the case $\tau_{\vartheta}(N)$, i.e., for statement $i i)$. The proof of statement $i$ ) is similar.

$(\Rightarrow)$ Since $u \in \tau_{\vartheta}(N)$, we know that $(N: u)$ is a $\vartheta$-Mathieu subspace of $\mathcal{A}$. Since $u \in N$, we have $1_{\mathcal{A}} \in(N: u)$. Then by Lemma 2.3, $(N: u)=\mathcal{A}$, whence $\mathcal{A} u \subseteq N$.

$(\Leftarrow)$ Since $\mathcal{A} u \subseteq N$, we have $u \in N$ and $(N: u)=\mathcal{A}$. Since $\mathcal{A}$ obviously is a $\vartheta$-Mathieu subspace of $\mathcal{A}$, we have $u \in \tau_{\vartheta}(N)$, whence $u \in N \cap \tau_{\vartheta}(N)$.

Recall that for each $R$-subspace $N$ of $\mathcal{M}$, we have set in Subsection $1.3 I_{N}$ to be the maximum $\mathcal{A}$-submodule of $N$, i.e., the unique $\mathcal{A}$ submodule of $\mathcal{M}$ which is maximum among all the $\mathcal{A}$-submodules of $\mathcal{M}$ contained in $N$. It is easy to see that $I_{N}$ always exists and is actually the same as the sum of all the $\mathcal{A}$-submodules of $\mathcal{M}$ contained in $N$. In particular, when $N$ itself is an $\mathcal{A}$-submodule, we have $I_{N}=N$.

Theorem 3.11. For every $R$-subspace $N$ of $\mathcal{M}$, we have

$$
I_{N}=N \cap \sigma_{\vartheta}(N)=N \cap \tau_{\vartheta}(N) \text {. }
$$

In particular, both $N \cap \tau_{\vartheta}(N)$ and $N \cap \sigma_{\vartheta}(N)$ are $\mathcal{A}$-submodules of $\mathcal{M}$. 
Proof: Note that the second equality in Eq. (3.11) follows directly from Lemma 3.10, So it suffices to show the first equality in Eq. (3.11).

We first show that $N \cap \sigma_{\vartheta}(N)$ is closed under the addition of $\mathcal{M}$. Let $u, v \in N \cap \sigma_{\vartheta}(N)$. Then by Lemma 3.10, $\left.i\right)$, we have $\mathcal{A} u \subseteq N$ and $\mathcal{A} v \subseteq N$. Hence, $\mathcal{A}(u+v) \subseteq \mathcal{A} u+\mathcal{A} v \subseteq N$ since $N$ is a $R$-subspace of $\mathcal{M}$. Then by Lemma 3.10, $i)$ again, we have $u+v \in N \cap \sigma_{\vartheta}(N)$.

Next, we show that $N \cap \sigma_{\vartheta}(N)$ is closed under the action of $\mathcal{A}$. Let $a \in \mathcal{A}$ and $u \in N \cap \sigma_{\vartheta}(N)$. By Lemma 3.10, $i$ ) we have $\mathcal{A} u \subseteq N$, whence $\mathcal{A}(a u) \subseteq \mathcal{A} u \subseteq N$. Then by Lemma 3.10, $i$ ) again, we have $a u \in N \cap \sigma_{\vartheta}(N)$.

Therefore, $N \cap \sigma_{\vartheta}(N)$ is indeed an $\mathcal{A}$-submodule of $\mathcal{M}$ (contained in $N)$. To show that $I_{N}=N \cap \sigma_{\vartheta}(N)$, it suffices to show that for each $\mathcal{A}$-submodule $H \subseteq N$, we have $H \subseteq N \cap \sigma_{\vartheta}(N)$.

Let $u \in H$. Since $H$ is an $\mathcal{A}$-submodule of $\mathcal{A}$, we have $\mathcal{A} u \subseteq H \subseteq N$. Then by lemma 3.10, $i), u \in N \cap \sigma_{\vartheta}(N)$. Hence $H \subseteq N \cap \sigma_{\vartheta}(N)$.

Next, we derive some consequences of Theorem 3.11.

Corollary 3.12. For every specification of $\vartheta$ and an $\mathcal{A}$-module $\mathcal{M}$, the following statements are equivalent:

1) $\mathcal{M}$ is an irreducible $\mathcal{A}$-module;

2) for every proper $R$-subspace $N$ of $\mathcal{M}$, we have $\sigma_{\vartheta}(N) \subseteq N^{c} \cup\{0\}$, where $N^{c}$ denotes the complement of $N$ in $\mathcal{M}$;

3) for every proper $R$-subspace $N$ of $\mathcal{M}$, we have $\tau_{\vartheta}(N) \subseteq N^{c} \cup\{0\}$.

Proof: If statement 1) holds, i.e., $\mathcal{M}$ is irreducible, then for every proper $R$-subspace $N$ of $\mathcal{M}$, we have $I_{N}=0$. By Eq. (3.11), we immediately have statements 2) and 3). Since by Lemma 3.3, $i$ ) $\sigma_{\vartheta}(N) \subseteq \tau_{\vartheta}(N)$ for every $R$-subspace $N$ of $\mathcal{M}$, we also have 3$) \Rightarrow 2$ ). Therefore, it suffices to show 2) $\Rightarrow 1$ ).

Assume that $\mathcal{M}$ is not irreducible. Let $N$ be a nonzero proper $\mathcal{A}$ submodule of $\mathcal{M}$. Then by Eq. (3.11), we have $N \cap \sigma_{\vartheta}(N)=I_{N}=N \neq$ 0 , which contradicts statement 2).

Corollary 3.13. Let $N$ be a $R$-subspace of $\mathcal{M}$. Then the following statements are equivalent:

1) $N \subseteq \sigma_{\vartheta}(N)$;

2) $N \subseteq \tau_{\vartheta}(N)$;

3) $N$ is an $\mathcal{A}$-submodule of $\mathcal{M}$.

Proof: $\quad$ Note that $N$ is an $\mathcal{A}$-submodule of $\mathcal{M}$ iff $I_{N}=N$. Then the corollary follows immediately from Eq. (3.11). 
Furthermore, for the case $\vartheta=$ "left", we can get $\sigma_{\vartheta}(N)$ and $\tau_{\vartheta}(N)$ explicitly under the equivalent conditions in the corollary above as follows.

Corollary 3.14. Let $\vartheta=$ "left" and $N$ be a $R$-subspace of $\mathcal{M}$. Then the following statements are equivalent:

1) $N$ satisfies one of the equivalent statements (with $\vartheta=$ "left") in Corollary 3.13 ;

2) $\sigma_{\vartheta}(N)=\mathcal{M}$

3) $\tau_{\vartheta}(N)=\mathcal{M}$.

Proof: Since statements 2) and 3) obviously imply respectively statements 1) and 2) in Corollary 3.13 , we have 2) $\Rightarrow 1$ ) and 3) $\Rightarrow 1$ ). Furthermore, by Lemma 3.3, $i$ ) we also have 2) $\Rightarrow 3$ ). Therefore, it suffices to show 1) $\Rightarrow 2$ ).

Assume that statement 3 ) in Corollary 3.13 holds, i.e., $N$ is a left $\mathcal{A}$-submodule of $\mathcal{M}$. Then it is easy to see that for any $u \in \mathcal{M},(N: u)$ is also a left ideal of $\mathcal{A}$, whence $u \in \sigma_{\vartheta}(N)$. Therefore, we have $\mathcal{M} \subseteq$ $\sigma_{\vartheta}(N)$, i.e., statement 2) holds.

Remark 3.15. When $\vartheta=$ "left", from Corollaries 3.13 and 3.14 we see that for each $R$-subspace $N$ of the $\mathcal{A}$-module $\mathcal{M}, N$ is an $\mathcal{A}$-submodule of $\mathcal{M}$ iff $N$ is a left Mathieu subspace of $\mathcal{M}$ with respect to all elements of $\mathcal{M}$. Therefore, the notion of left Mathieu subspaces of $\mathcal{A}$-modules in the sense above does generalize the notion of $\mathcal{A}$-submodules.

Next, we study some functorial properties of the sets of $\vartheta$-stable and $\vartheta$-quasi-stable elements of $R$-subspaces of $\mathcal{A}$-modules.

Proposition 3.16. Let $\mathcal{M}, \mathcal{N}$ be $\mathcal{A}$-modules and $\phi: \mathcal{M} \rightarrow \mathcal{N}$ a homomorphism of $\mathcal{A}$-modules. Then for every $R$-subspace $H$ of $\mathcal{N}$, we have

$$
\begin{aligned}
\phi^{-1}\left(\sigma_{\vartheta}(H)\right) & =\sigma_{\vartheta}\left(\phi^{-1}(H)\right), \\
\phi^{-1}\left(\tau_{\vartheta}(H)\right) & =\tau_{\vartheta}\left(\phi^{-1}(H)\right) .
\end{aligned}
$$

Proof: Note first that for each $u \in \mathcal{M}$, it is easy to check that the following equation always holds:

$$
(H: \phi(u))=\left(\phi^{-1}(H): u\right) .
$$

Then for each $u \in \mathcal{M}$, we have that $u \in \tau_{\vartheta}\left(\phi^{-1}(H)\right)$, iff $\left(\phi^{-1}(H): u\right)$ is a $\vartheta$-Mathieu subspace of $\mathcal{A}$, iff $(H: \phi(u))$ by Eq. (3.14) is a $\vartheta$ Mathieu subspace of $\mathcal{A}$, iff $\phi(u) \in \tau_{\vartheta}(H)$, iff $u \in \phi^{-1}\left(\tau_{\vartheta}(H)\right)$. Hence, Eq. (3.13) follows. The proof of Eq. (3.12) is similar. 
Next we derive some consequences of Proposition 3.16.

Corollary 3.17. Let $a \in \mathcal{A}$ be a central element of $\mathcal{A}$, i.e., a commutes with all elements of $\mathcal{A}$. Then for every $R$-subspace $N$ of $\mathcal{M}$, we have

$$
\begin{aligned}
& a^{-1} \sigma_{\vartheta}(N)=\sigma_{\vartheta}\left(a^{-1} N\right), \\
& a^{-1} \tau_{\vartheta}(N)=\tau_{\vartheta}\left(a^{-1} N\right) .
\end{aligned}
$$

Proof: Let $\mu_{a}: \mathcal{M} \rightarrow \mathcal{M}$ be the $R$-linear map defined by the action of $a$ on $\mathcal{M}$. Since $a$ is a central element of $\mathcal{A}$, the map $\mu_{a}$ is an endomorphism of the $\mathcal{A}$-module $\mathcal{M}$. Then the corollary follows immediately from Proposition 3.16 with $\phi=\mu_{a}$.

Corollary 3.18. Let $V$ be an $\mathcal{A}$-submodule of $\mathcal{M}$ and $N$ a $R$-subspace of $\mathcal{M}$. Then we have

$$
\begin{gathered}
V \cap \sigma_{\vartheta}(N)=\sigma_{\vartheta}(V \cap N), \\
V \cap \tau_{\vartheta}(N)=\tau_{\vartheta}(V \cap N),
\end{gathered}
$$

where $\sigma_{\vartheta}(V \cap N)$ (resp., $\tau_{\vartheta}(V \cap N)$ ) is the set of $\vartheta$-stable (resp., $\vartheta$ quasi-stable) elements of $V \cap N$ as a $R$-subspace of the $\mathcal{A}$-module $V$.

Proof: Let $\iota: V \rightarrow \mathcal{M}$ be the embedding of $V$ into $\mathcal{M}$. Note that for every subset $S$ of $\mathcal{M}$, we have $\iota^{-1}(S)=V \cap S$. Then the corollary follows immediately from this observation and Proposition 3.16 with $\phi=\iota$.

Corollary 3.19. In the same setting as in Proposition 3.16, let $u \in \mathcal{M}$ and $v \in \mathcal{N}$ such that $\phi(u)=v$. Then $H$ is $\vartheta$-Mathieu subspace of $\mathcal{N}$ with respect to $v \in \mathcal{N}$ iff $\phi^{-1}(H)$ is $\vartheta$-Mathieu subspace of $\mathcal{M}$ with respect to $u \in \mathcal{M}$.

Proof: $\quad H$ is a $\vartheta$-Mathieu subspace of $\mathcal{N}$ with respect to $v$, iff $\phi(u)=$ $v \in \tau_{\vartheta}(H)$, iff $u \in \phi^{-1}\left(\tau_{\vartheta}(H)\right)$, iff $u \in \tau_{\vartheta}\left(\phi^{-1}(H)\right)$ (by Eq. (3.13)), iff $\phi^{-1}(H)$ is a $\vartheta$-Mathieu subspace of $\mathcal{M}$ with respect to $u$.

Remark 3.20. A fundamental property of submodules is that they are closed under the pull-backs of homomorphisms of modules. It is easy to see that Corollary 3.19 generalizes this property of submodules to $\vartheta$-Mathieu subspaces of modules of associative algebras. Furthermore, Corollary 3.19 can also be viewed as a generalization of the similar property of $\vartheta$-Mathieu subspaces of associative algebras given in Proposition 2.6 if we view the $R$-algebra $\mathcal{B}$ in Proposition 2.6 as an $\mathcal{A}$-module via the $R$-algebra homomorphism $\phi: \mathcal{A} \rightarrow \mathcal{B}$, and choose $u=1_{\mathcal{A}}$ and $v=1_{\mathcal{B}}$. 
Next, applying Proposition 3.16 to the quotient maps of associative algebras $\mathcal{A}$, we get the following corollary, which can be viewed as a generalization of Proposition 2.5.

Corollary 3.21. Let $\mathcal{M}$ be an $\mathcal{A}$-module and $V$ an $\mathcal{A}$-submodule of $\mathcal{M}$. Denote by $\pi: \mathcal{M} \rightarrow \mathcal{M} / V$ the quotient map. Then for every $u \in \mathcal{M}$ and $R$-subspace $N$ of $\mathcal{M}$ such that $V \subseteq N$, we have

$$
\begin{gathered}
\sigma_{\vartheta}(N)=\pi^{-1}\left(\sigma_{\vartheta}(N / V)\right), \\
\tau_{\vartheta}(N)=\pi^{-1}\left(\tau_{\vartheta}(N / V)\right), \\
\pi\left(\sigma_{\vartheta}(N)\right)=\sigma_{\vartheta}(N / V), \\
\pi\left(\tau_{\vartheta}(N)\right)=\tau_{\vartheta}(N / V) .
\end{gathered}
$$

Proof: Since $V \subseteq N$, we have $\pi^{-1}(N / V)=N$. Then by applying Proposition 3.16 with $\phi=\pi$, we see that Eqs. (3.19) and (3.20) follow immediately from Eqs. (3.12) and (3.13), respectively. Furthermore, since $\pi$ is surjective, Eqs. (3.21) and (3.22) follow respectively from Eqs. (3.19) and (3.20).

Finally, we conclude this section with the following variation of Proposition 3.16.

Proposition 3.22. Let $\psi: \mathcal{A} \rightarrow \mathcal{B}$ be a R-algebra homomorphism. Then for every $R$-subspace $J$ of $\mathcal{B}$, we have

$$
\begin{aligned}
& \psi^{-1}\left(\sigma_{\vartheta}(J)\right) \subseteq \sigma_{\vartheta}\left(\psi^{-1}(J)\right), \\
& \psi^{-1}\left(\tau_{\vartheta}(J)\right) \subseteq \tau_{\vartheta}\left(\psi^{-1}(J)\right) .
\end{aligned}
$$

Furthermore, if $\psi$ is surjective, then the equalities in the both equations above hold.

Remark 3.23. The reason that we do not always have equality in Eq. (3.24) (in contrast to Eq. (3.13)) is because that the sets $\tau_{\vartheta}(J)$ and $\tau_{\vartheta}\left(\psi^{-1}(J)\right)$ of quasi-stable elements of $J$ and $\psi^{-1}(J)$, respectively, are defined with respect to the different algebras $\mathcal{B}$ and $\mathcal{A}$. This is also the case for Eq. (3.23).

Proof of Proposition 3.22: Note first that for each $a \in \mathcal{A}$, it is easy to check directly that the following equation holds:

$$
\psi^{-1}(J: \psi(a))=\left(\psi^{-1}(J): a\right) .
$$

Note also that the sets $(J: \psi(a))$ and $\left(\psi^{-1}(J): a\right)$ in the equation above are defined with respect to the (different) $R$-algebras $\mathcal{B}$ and $\mathcal{A}$, respectively. 
Now, for each $a \in \psi^{-1}\left(\tau_{\vartheta}(J)\right)$, we have $\psi(a) \in \tau_{\vartheta}(J)$, whence $(J$ : $\psi(a))$ is a $\vartheta$-Mathieu subspace of the $R$-algebra $\mathcal{B}$. Then by Eq. (3.25) and Proposition 2.6, $\left(\psi^{-1}(J): a\right)$ is a $\vartheta$-Mathieu subspace of $\mathcal{A}$. Hence, we have $a \in \tau_{\vartheta}\left(\psi^{-1}(J)\right)$, whence Eq. (3.24) follows. Eq. (3.23) can be proved similarly by using Eq. (3.25) and the fact that $\vartheta$-ideals are closed under the pulling-back by $R$-algebra homomorphisms.

Next we assume that $\psi$ is surjective and show that the equality in Eq. (3.24) does hold. The equality in Eq. (3.23) can be proved similarly.

Let $a \in \tau_{\vartheta}\left(\psi^{-1}(J)\right)$. Then $\left(\psi^{-1}(J): a\right)$ is a $\vartheta$-Mathieu subspace of $\mathcal{A}$. We need to show $a \in \psi^{-1}\left(\tau_{\vartheta}(J)\right)$ or equivalently, $(J: \psi(a))$ is a $\vartheta$-Mathieu subspace of $\mathcal{B}$.

Let $I$ be the kernel of the $R$-homomorphism $\psi$. Then $I$ is an ideal of $\mathcal{A}$. By identifying the $R$-algebra $\mathcal{B}$ with the quotient $R$-algebra $\mathcal{A} / I$ via the induced $R$-algebra isomorphism $\bar{\psi}: \mathcal{A} / I \stackrel{\sim}{\rightarrow} \mathcal{B}$, we may assume that $\mathcal{B}=\mathcal{A} / I$ and the $R$-algebra homomorphism $\psi$ is the quotient map $\pi: \mathcal{A} \rightarrow \mathcal{A} / I$.

With the setting above, it is easy to check that we have

$$
I \subseteq\left(\psi^{-1}(J): a\right)
$$

Combining the equation above with Eq. (3.25), we also have

$$
\left(\psi^{-1}(J): a\right) / I=(J: \psi(a)) .
$$

Then with Eqs. (3.26) and (3.27) above, applying Proposition 2.5 to the $\vartheta$-Mathieu subspace $\left(\psi^{-1}(J): a\right)$ of $\mathcal{A}$, we see that $(J: \psi(a))$ is indeed a $\vartheta$-Mathieu subspace of $\mathcal{B}$.

\section{Quasi-Stable Modules and Quasi-Stable Algebras}

Let $R, \mathcal{A}$ and $\mathcal{M}$ be fixed as before. We first introduce the following notions.

Definition 4.1. An $\mathcal{A}$-module $\mathcal{M}$ is said to be $\vartheta$-quasi-stable (resp., $\vartheta$-stable) if for every $R$-subspace $N$ of $\mathcal{M}$, we have $N^{c} \subseteq \tau_{\vartheta}(N)$ (resp., $\left.N^{c} \subseteq \sigma_{\vartheta}(N)\right)$, where $N^{c}$ denotes the complement of the subset $N$ in $\mathcal{M}$.

Note that by Lemma [3.3, $i$ ), we see that the $\vartheta$-stableness implies $\vartheta$-quasi-stableness.

From Definition 4.1, Theorem 3.11 and also the observation above, it is easy to see that the following lemma holds.

Lemma 4.2. $i$ ) If $\mathcal{M}$ is a $\vartheta$-quasi-stable $\mathcal{A}$-module, then for any $R$ subspace $N$ of $\mathcal{M}$, we have

$$
\tau_{\vartheta}(N)=I_{N} \cup N^{c} .
$$


ii) If $\mathcal{M}$ is a $\vartheta$-stable $\mathcal{A}$-module, then for any $R$-subspace $N$ of $\mathcal{M}$, we have

$$
\sigma_{\vartheta}(N)=\tau_{\vartheta}(N)=I_{N} \cup N^{c} .
$$

When the $R$-algebra $\mathcal{A}$ itself is viewed as a left $\mathcal{A}$-module (in the canonical way), then the next lemma shows that Definitions 2.7 and 4.1 actually coincide.

Lemma 4.3. For every $R$-algebra $\mathcal{A}$, it is $\vartheta$-quasi-stable (resp., $\vartheta$ stable) as a $R$-algebra iff it is $\vartheta$-quasi-stable (resp., $\vartheta$-stable) as a left $\mathcal{A}$-module.

Proof: We just give a proof here for the $\vartheta$-quasi-stable case. The $\vartheta$-stable case can be proved similarly.

$(\Rightarrow)$ Let $J$ be a $R$-subspace of $\mathcal{A}$ and $a \in J^{c}$. Then $1 \notin(J: a)$ and the $R$-subspace $(J: a)$ is a $\vartheta$-Mathieu subspace of $\mathcal{A}$, for $\mathcal{A}$ is a $\vartheta$-quasistable $R$-algebra, whence $a \in \tau_{\vartheta}(J)$. Therefore, we have $J^{c} \subseteq \tau_{\vartheta}(J)$. Hence $\mathcal{A}$ as a left $\mathcal{A}$-module is also $\vartheta$-quasi-stable.

$(\Leftarrow)$ Let $J$ be a $R$-subspace of $\mathcal{A}$ with $1 \notin J$. Since $\mathcal{A}$ as a left $\mathcal{A}$-module is $\vartheta$-quasi-stable, we have $J^{c} \subseteq \tau_{\vartheta}(J)$. Since $1 \in J^{c}$, we have $1 \in \tau_{\vartheta}(J)$, whence $J=(J: 1)$ is a $\vartheta$-Mathieu subspace of $\mathcal{A}$. Therefore, $\mathcal{A}$ as a $R$-algebra is also $\vartheta$-quasi-stable.

Actually, the following more general result also holds.

Proposition 4.4. For every $R$-algebra $\mathcal{A}$, the following two statements are equivalent to each other.

1) $\mathcal{A}$ as a $R$-algebra is $\vartheta$-quasi-stable (resp., $\vartheta$-stable).

2) Every left $\mathcal{A}$-module $\mathcal{M}$ is $\vartheta$-quasi-stable (resp., $\vartheta$-stable).

Proof: 2) $\Rightarrow$ 1) follows from Lemma 4.3, 1) $\Rightarrow$ 2) can be shown by a similar argument as in the proof of the $(\Rightarrow)$ part of Lemma 4.3, at least for the $\vartheta$-quasi-stable case. The $\vartheta$-stable case can also be proved similarly.

Combining the proposition above with Lemma 4.2, Proposition 2.8, Theorem 2.10 and Proposition 2.11, we immediately have the following corollary.

Corollary 4.5. Let $\mathcal{A}$ be a $R$-algebra as in Proposition 2.8 or a $K$ algebra satisfying one of the equivalent statements in Proposition 2.11, or $\mathcal{A} \simeq K \dot{+} K$. Then all left $\mathcal{A}$-modules $\mathcal{M}$ are $\vartheta$-quasi-stable. Consequently, for any $R$-subspace $N$ of $\mathcal{M}$, we have $\tau_{\vartheta}(N)=I_{N} \cup N^{c}$, which is actually independent on the specifications of $\vartheta$. 
Corollary 4.6. Let $\mathcal{A}$ be an algebraic field extension of a field $K$ or more generally, an algebraic division algebra over $K$. Then for every $K$-subspace $J$ of $\mathcal{A}$, the following two statements hold.

i) If $J=0$ or $\mathcal{A}$, then we have

$$
\sigma_{\vartheta}(J)=\tau_{\vartheta}(J)=\mathcal{A} .
$$

ii) If $J$ is nonzero and proper, then we have

$$
\begin{aligned}
\sigma_{\vartheta}(J) & =\{0\}, \\
\tau_{\vartheta}(J) & =J^{c} \cup\{0\} .
\end{aligned}
$$

Proof: Note first that for every $a \in \mathcal{A}$, we have

$$
(\mathcal{A}: a)=\mathcal{A} \text {. }
$$

Since $\mathcal{A}$ has no zero-divisors, we also have

$$
(0: a)= \begin{cases}\mathcal{A} & \text { if } a=0 \\ 0 & \text { if } a \neq 0\end{cases}
$$

Then it is easy to see that Eq. (4.3) follows immediately from the two equations above.

To show Eq. (4.4), assume that $J$ is nonzero and proper. Note first that for each $0 \neq a \in \mathcal{A}$ and $0 \neq b \in J$, we have $0 \neq b a^{-1} \in(J: a)$, whence $(J: a) \neq 0$. Moreover, $(J: a) \neq \mathcal{A}$ either, for otherwise we would have $\mathcal{A}=\mathcal{A} a \subseteq J$, which contradicts our assumption that $J$ is proper. Therefore, $(J: a)$ is also a nonzero proper $K$-subspace of $\mathcal{A}$.

On the other hand, since $\mathcal{A}$ has no nonzero proper $\vartheta$-ideals (for every nonzero element of $\mathcal{A}$ is invertible), the $K$-subspace $(J: a)$ cannot be a $\vartheta$-ideal of $\mathcal{A}$. Hence we have $a \notin \sigma_{\vartheta}(J)$ for all $0 \neq a \in \mathcal{A}$, whence Eq. (4.4) follows.

To show Eq. (4.5), note first that by Theorem 2.10 and Proposition 2.11, $\mathcal{A}$ is a $\vartheta$-quasi-stable $K$-algebra. Then by Lemma 4.3 or Proposition 4 .4, $\mathcal{A}$ as a left $\mathcal{A}$-module is also $\vartheta$-quasi-stable. Therefore, by Eq. (4.1) we have $\tau_{\vartheta}(J)=I_{J} \cup J^{c}$, where $I_{J}$ is the (unique) left ideal of $\mathcal{A}$ which is maximum among all the left ideals of $\mathcal{A}$ contained in $J$.

On the other hand, since $\mathcal{A}$ has no nonzero proper left ideals (as already pointed out above), we have $I_{J}=0$, whence Eq. (4.5) follows.

Remark 4.7. Let $\mathcal{A}$ be as in Corollary 4.6. Then $\mathcal{A}$ as a left $\mathcal{A}$-module is irreducible, since every nonzero element of $\mathcal{A}$ is invertible. Therefore, Corollary 4.6 provides a family of irreducible $\mathcal{A}$-modules $\mathcal{M}$ such that $\tau_{\vartheta}(J) \neq 0$ for all proper $K$-subspaces $J$ of $\mathcal{M}$. 
Finally, we conclude this section with the following open problem which, we believe, is worthy to be much further investigated.

Problem 4.8. Classify all $\vartheta$-stable or $\vartheta$-quasi-stable modules for some "nice" associative algebras, say, for semi-simple algebras or Noetherian algebras.

\section{Two Cases for Modules of Matrix Algebras over Fields}

In this section, we fix an arbitrary field $K$ and let $M_{n}(K)(n \geq 1)$ denote the matrix algebra of $n \times n$ matrices with entries in $K$.

We first consider the sets $\sigma_{\vartheta}(N)$ and $\tau_{\vartheta}(N)$ for $K$-subspaces $N$ of the $M_{n}(K)$-module $K^{n}$ (with the standard left action).

Proposition 5.1. Let $n \geq 2$ and $N$ a $K$-subspace of the $M_{n}(K)$ module $K^{n}$. Then the following statements hold.

i) If $N=K^{n}$, we have

$$
\sigma_{\vartheta}(N)=\tau_{\vartheta}(N)=K^{n} .
$$

ii) If $N=0$, we have

$$
\sigma_{\vartheta}(N)=\tau_{\vartheta}(N)= \begin{cases}K^{n} & \text { if } \vartheta=\text { "left" } ; \\ 0 & \text { otherwise. }\end{cases}
$$

iii) If $N$ is nonzero and proper, we have

$$
\sigma_{\vartheta}(N)=\tau_{\vartheta}(N)=0 .
$$

Note that the case $n=1$ has been covered by Corollary 4.6 in the previous section. More precisely, in this case Eq. (5.1) still holds but not Eq. (5.2). Instead, we have $\sigma_{\vartheta}(0)=\tau_{\vartheta}(0)=K$ for every specification of $\vartheta$.

In order to prove the proposition above, we first need to show the following lemma.

Lemma 5.2. Let $n \geq 2$ and $u, v \in K^{n}$ such that $u$ and $v$ are $K$-linearly independent with each other. Then the following two statements hold:

$i)$ there exists an idempotent $E_{1} \in M_{n}(K)$ such that $E_{1} u=v$ and $E_{1} v=v$;

ii) there exists an idempotent $E_{2} \in M_{n}(K)$ such that $E_{2} u=0$ and $E_{2} v=u+v$.

Proof: We identify $M_{n}(K)$ with the $K$-algebra $\operatorname{End}_{K}\left(K^{n}\right)$ of $K$ linear endomorphisms of $K^{n}$ via the standard basis of $K^{n}$.

Let $\left\{v_{1}, v_{2}, \ldots, v_{n}\right\}$ be a $K$-linear basis of $K^{n}$ such that $u=v_{1}$ and $v=v_{2}$. Let $E_{1}$ be the $K$-linear map such that $E_{1} v_{1}=v_{2}$ and $E_{1} v_{i}=v_{i}$ 
for all $2 \leq i \leq n$. Then it is easy to check that $E_{1}^{2}=E_{1}$, whence $i$ ) follows.

To show $i i$ ), let $E_{2}$ be the $K$-linear map such that $E_{2} v_{1}=0 ; E_{2} v_{2}=$ $v_{1}+v_{2}$ and $E_{2} v_{i}=v_{i}$ for all $3 \leq i \leq n$. Then it is easy to check that $E_{2}^{2}=E_{2}$, whence $i$ ) follows.

Proof of Proposition 5.1: Note first that Eq. (5.1) and also the case $\vartheta=$ "left" of Eq. (5.2) follow directly from Lemma 3.2.

To show the case $\vartheta=$ "right" of Eq. (5.2), by Lemma 3.3, $i$ ), it suffices to show $\tau_{\vartheta}(0)=0$ for $\vartheta=$ "right". More explicitly, it suffices to show that the annihilator $(0: u)$ for each $0 \neq u \in K^{n}$ is not a right Mathieu subspace of $M_{n}(K)$.

We fix an arbitrary $0 \neq u \in K^{n}$ and pick up a nonzero $v \in K^{n}$ such that $u$ and $v$ are $K$-linearly independent. Applying Lemma 5.2 to the vectors $u$ and $v$, and letting $E_{i}(i=1,2)$ be the idempotents in the same lemma, we have $E_{2} \in(0: u)$ but $E_{2} E_{1} \notin(0: u)$, for $E_{2} E_{1} u=E_{2} v=u+v \neq 0$. Hence, the right ideal of $M_{n}(K)$ generated by the idempotent $E_{2}$ is not contained in $(0: u)$. Then by Theorem 2.9, $(0: u)$ is not a right Mathieu subspace of $M_{n}(K)$. Therefore, the case $\vartheta=$ "right" of Eq. (5.2) holds, whence so do the cases $\vartheta=$ "pretwo-sided" and $\vartheta=$ "two-sided".

To show Eq. (5.3), by Lemma 3.3, $i$ ), it suffices to show $\tau_{\vartheta}(N)=0$. Moreover, since $K^{n}$ is an irreducible $M_{n}(K)$-module, by Corollary 3.12 , we have $\tau_{\vartheta}(N) \subseteq N^{c} \cup\{0\}$. Therefore, it suffices to show

$$
N^{c} \cap \tau_{\vartheta}(N)=\emptyset
$$

Now, let $u \in N^{c}$ and choose any $0 \neq v \in N$. Note that $u$ and $v$ are $K$-linearly independent. Applying Lemma 5.2 to $u$ and $v$, and letting $E_{i}(i=1,2)$ be the idempotents in the same lemma, we have $E_{1}, E_{2} \in(N: u)$.

Let $A \in M_{n}(K)$ such that $A u=v$ and $A v=u$ (Note that by identifying $M_{n}(K)$ with $\operatorname{End}_{K}\left(K^{n}\right)$, as in the proof of Lemma 5.2, it is easy to see that such a matrix $A$ does exist). Then we have $A E_{1} u=u \notin N$. Hence, $A E_{1} \notin(N: u)$ and by Theorem 2.9, $(N: u)$ is not a left Mathieu subspace of $M_{n}(K)$. On the other hand, we also have $E_{2} A u=E_{2} v=u+v \notin N$. Hence, $E_{2} A \notin(N: u)$, and by Theorem [2.9, $(N: u)$ is not a right Mathieu subspace of $M_{n}(K)$ either. Consequently, $u \notin \tau_{\vartheta}(N)$ when $\vartheta=$ "left" or "right", and hence the same when $\vartheta=$ "pre-two-sided" and $\vartheta=$ "two-sided". Therefore, Eq. (5.4) does hold for all specifications of $\vartheta$. 
Next, we consider the sets $\sigma_{\vartheta}(J)$ and $\tau_{\vartheta}(J)$ for co-dimension one $K$-subspaces $J$ of $M_{n}(K)$ as a left $M_{n}(K)$-module. First, let's fix the following notations.

We denote by $I_{n}$ the identity $n \times n$ matrix and $\operatorname{Tr}: M_{n}(K) \rightarrow K$ the trace function of $M_{n}(K)$. Furthermore, we set

$$
H_{X}:=\left\{A \in M_{n}(K) \mid \operatorname{Tr}(A X)=0\right\} .
$$

For simplicity, we also denote $H_{I_{n}}$ by $H$, i.e., $H$ is the co-dimension one $K$-subspace of the trace-zero matrices of $M_{n}(K)$.

Note that the trace function $\operatorname{Tr}: M_{n}(K) \rightarrow K$ induces the following non-singular $K$-bilinear form of $M_{n}(K)$ :

$$
\begin{aligned}
(\cdot, \cdot): M_{n}(K) \times M_{n}(K) & \rightarrow K \\
(Y, \quad X) & \rightarrow \operatorname{Tr}(Y X) .
\end{aligned}
$$

Let $X, Y \in M_{n}(K)$. We denote by $X \sim Y$ if $X=\alpha Y$ for some $\alpha \in K^{\times}$. Note that by Eq. (5.5) and the non-singularity of the $K$ bilinear form in Eq. (5.6), it is easy to check that

$$
H_{X}=H_{Y} \Leftrightarrow X \sim Y \text {. }
$$

In particular, we have

$$
\begin{aligned}
H_{X}=M_{n}(K) & \Leftrightarrow X=0, \\
H_{X}=H & \Leftrightarrow X \sim I_{n} .
\end{aligned}
$$

Furthermore, it is also easy to check (e.g., see Lemma 5.2 in [Z6]) that every co-dimension one $K$-subspace of $M_{n}(K)$ has the form $H_{X}$ for some $0 \neq X \in M_{n}(K)$ and the matrix $X$ by Eq. (5.7) is unique up to nonzero scalar multiplications.

With the notations fixed above, we can state our second main result of this section as follows.

Proposition 5.3. Let $K$ be a field and $n \geq 2$. Then for each $X \in$ $M_{n}(K)$, the following statements hold.

i) If char. $K=0$ or char. $K=p>n$, we have

$$
\begin{aligned}
\sigma_{\vartheta}\left(H_{X}\right) & =(0: X)=\left\{Y \in M_{n}(K) \mid Y X=0\right\} . \\
\tau_{\vartheta}\left(H_{X}\right) & =\left\{Y \in M_{n}(K) \mid Y X=0 \text { or } Y X \sim I_{n}\right\} .
\end{aligned}
$$

ii) If char. $K=p>0$ and $p \leq n$, then

$$
\sigma_{\vartheta}\left(H_{X}\right)=\tau_{\vartheta}\left(H_{X}\right)=(0: X) .
$$

Note that the proposition above requires $n \geq 2$. The case $n=1$ has been covered by Corollary 4.6 in Section 4, Note also that for all $n \geq 1$ and $X \in M_{n}(K)$, by Corollary 4.6 and the proposition above the sets 
$\sigma_{\vartheta}\left(H_{X}\right)$ and $\tau_{\vartheta}\left(H_{X}\right)$ are actually independent on the specifications of $\vartheta$.

In order to prove the proposition above, we need the following theorem proved in [Z6] on the co-dimension one $\vartheta$-Mathieu subspaces of $M_{n}(K)$.

Theorem 5.4. Let $K$ be a field and $n \geq 1$. Then for every fixed specification of $\vartheta$, the following two statements hold.

$i)$ If char. $K=0$ or char. $K=p>n$, then $H$ is the only codimension one $\vartheta$-Mathieu subspace of $M_{n}(K)$.

ii) If char. $K=p>0$ and $p \leq n$, then $M_{n}(K)$ has no co-dimension one $\vartheta$-Mathieu subspace.

Proof of Proposition 5.3: Let $Y \in M_{n}(K)$. Then by the definition

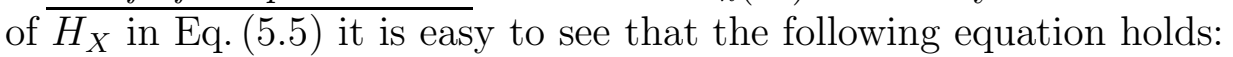

$$
\left(H_{X}: Y\right)=H_{Y X} \text {. }
$$

Assume first $Y X=0$, i.e., $Y \in(0: X)$. Then by the equation above and Eq. (5.8), we have $\left(H_{X}: Y\right)=M_{n}(K)$. Since $M_{n}(K)$ is obviously a $\vartheta$-ideal of $M_{n}(K)$, we have $Y \in \sigma_{\vartheta}\left(H_{X}\right)$. Consequently, we have $(0: X) \subseteq \sigma_{\vartheta}\left(H_{X}\right)$. By Lemma 3.3, $\left.i\right)$, we also have

$$
(0: X) \subseteq \sigma_{\vartheta}\left(H_{X}\right) \subseteq \tau_{\vartheta}\left(H_{X}\right)
$$

Assume $Y X \neq 0$ and $Y X \not I_{n}$. Then by Eq. (5.9), we have $H_{Y X} \neq$ $H$, and by Theorem 5.4 and Eq. (5.13), $\left(H_{X}: Y\right)$ is not a $\vartheta$-Mathieu subspace of $M_{n}(K)$ and hence, not a $\vartheta$-ideal of $M_{n}(K)$ either. Therefore, in this case we have

$$
Y \notin \tau_{\vartheta}\left(H_{X}\right) \text { and } Y \notin \sigma_{\vartheta}\left(H_{X}\right) \text {. }
$$

Now, assume $Y X \sim I_{n}$. Then by Eqs. (5.9) and (5.13), we have $\left(H_{X}: Y\right)=H$. Furthermore, by Theorem 5.4 we see that $\left(H_{X}: Y\right)$ is a $\vartheta$-Mathieu subspace of $M_{n}(K)$, i.e., $Y \in \tau_{\vartheta}\left(H_{X}\right)$, iff the condition of the statement $i$ ) holds.

From the arguments above, it is easy to see that Eq. (5.12) for $\tau_{\vartheta}\left(H_{X}\right)$ and also Eq. (5.11) indeed hold, i.e., the proposition holds for the set $\tau_{\vartheta}\left(H_{X}\right)$. Furthermore, by Eq. (5.14) and Eq. (5.12) for $\tau_{\vartheta}\left(H_{X}\right)$, we also have Eq. (5.12) for $\sigma_{\vartheta}\left(H_{X}\right)$.

Finally, to show Eq. (5.10), by Eqs. (5.13)-(5.15) it suffices to show that the co-dimension one $K$-subspace $H$ is not a left or right ideal of $M_{n}(K)$. But this can be easily checked under the condition $n \geq 2$. Therefore, the proposition follows. 
One consequence of Proposition 5.3 is the following corollary, which can also be proved directly by using the non-singularity of the paring in Eq. (5.6) .

Corollary 5.5. Let $K$ be a field and $n \geq 1$. For each $X \in M_{n}(K)$, denote by $I_{X}$ (resp., $\left.J_{X}\right)$ the unique left (resp., right) ideal of $M_{n}(K)$ which is maximum among all the left (resp., right) ideals of $M_{n}(K)$ contained in $H_{X}$. Then we have

$$
\begin{aligned}
& I_{X}=(0: X)=\left\{Y \in M_{n}(K) \mid Y X=0\right\}, \\
& J_{X}=\left\{Y \in M_{n}(K) \mid X Y=0\right\} .
\end{aligned}
$$

Proof: The case $n=1$ can be checked easily. So we assume $n \geq 2$. Note that by Proposition 5.3 we have

$$
\sigma_{\vartheta}\left(H_{X}\right)=\left\{Y \in M_{n}(K) \mid Y X=0\right\} .
$$

Then viewing $M_{n}(K)$ as a left $M_{n}(K)$-module and applying Theorem 3.11, we see that Eq. (5.16) follows from Eq. (5.18) for the case $\vartheta=$ "left".

To show Eq. (5.17), we first consider the transpose map

$$
\begin{aligned}
t: M_{n}(K) & \rightarrow M_{n}(K) \\
Y & \rightarrow Y^{t}
\end{aligned}
$$

where $Y^{t}$ denotes the transpose of the matrix $Y$.

Note that the map $t$ is an anti-involution of $K$-algebra $M_{n}(K)$, i.e., $t^{2}=\mathrm{id}$, the identity map of $M_{n}(K)$, and $t(X Y)=t(Y) t(X)$ for all $X, Y \in M_{n}(K)$. Therefore, $t$ induces an one-to-one correspondence between the set of all left ideals $J$ of $M_{n}(K)$ and the set of all right ideals of $M_{n}(K)$ via $J \leftrightarrow t(J)$.

Furthermore, for all $X, Y \in M_{n}(K)$, we also have

$$
\operatorname{Tr}(Y X)=\operatorname{Tr}\left((Y X)^{t}\right)=\operatorname{Tr}\left(X^{t} Y^{t}\right)=\operatorname{Tr}\left(Y^{t} X^{t}\right) .
$$

From Eq. (5.5) and the equation above, it is easy to see that for each $X \in M_{n}(K)$, we have

$$
t\left(H_{X}\right)=H_{X^{t}}
$$

Now, by Eq. (5.16) with $X$ replaced by $X^{t}$, we have

$$
I_{X^{t}}=\left(0: X^{t}\right)=\left\{Y \in M_{n}(K) \mid Y X^{t}=0\right\} .
$$

Then by the last two equations above, we have

$$
\begin{aligned}
J_{X} & =t^{-1}\left(I_{X^{t}}\right)=t\left(I_{X^{t}}\right)=\left\{Y^{t} \in M_{n}(K) \mid Y X^{t}=0\right\} \\
& =\left\{Y \in M_{n}(K) \mid Y^{t} X^{t}=0\right\} \\
& =\left\{Y \in M_{n}(K) \mid(X Y)^{t}=0\right\}
\end{aligned}
$$




$$
=\left\{Y \in M_{n}(K) \mid X Y=0\right\} .
$$

Hence, we get Eq. (5.17).

\section{Two Cases for Modules of Polynomial Algebras over Fields}

Let $K$ be a field and $z=\left(z_{1}, z_{2}, \ldots, z_{n}\right) n$ commutative free variables. In this section, we consider the sets $\sigma_{\vartheta}(N)$ and $\tau_{\vartheta}(N)$ of the stable elements and quasi-stable elements, respectively, of two families of codimension one $K$-subspaces $N$ of the polynomial algebra $K[z]$ (as a $K[z]$-module).

Note that $K[z]$ is commutative. The sets $\sigma_{\vartheta}(N)$ and $\tau_{\vartheta}(N)$ are actually independent on the specifications of $\vartheta$. Therefore, we simply denote them by $\sigma(N)$ and $\tau(N)$, respectively. Furthermore, we also need to fix the following notations.

For any $\ell \geq 1$ and $\alpha=\left(\alpha_{1}, \alpha_{2}, \ldots, \alpha_{\ell}\right) \in K^{\ell}$, we denote by $S_{\alpha}$ the set of $1 \leq i \leq \ell$ such that $\alpha_{i} \neq 0$. We let $\Omega_{\ell}$ be the set of $\alpha=\left(\alpha_{1}, \alpha_{2}, \ldots, \alpha_{\ell}\right) \in K^{\ell}$, which satisfies the following property:

(*) for any non-empty $C \subseteq S_{\alpha}$, we have $\sum_{i \in C} \alpha_{i} \neq 0$.

Note that $S_{\alpha}=\emptyset$ iff $\alpha=0$, and in this case the property $(*)$ above is satisfied vacuously. Therefore, we have $0 \in \Omega_{\ell}$.

Let $\alpha \in K^{\ell}$ (as above) and $B=\left\{u_{1}, u_{2}, \ldots, u_{\ell}\right\} \subseteq K^{n}$ such that $u_{i} \neq u_{j}$ for all $1 \leq i \neq j \leq \ell$. For each $f \in K[z]$, we set

$$
\alpha_{f, B}:=\left(\alpha_{1} f\left(u_{1}\right), \alpha_{2} f\left(u_{2}\right), \ldots, \alpha_{\ell} f\left(u_{\ell}\right)\right) \in K^{\ell} .
$$

Furthermore, we also introduce the following $K$-subspace of the polynomial algebra $K[z]$ :

$$
N_{B, \alpha}:=\left\{f(z) \in K[z] \mid \sum_{i=1}^{\ell} \alpha_{i} f\left(u_{i}\right)=0\right\} .
$$

Note that $N_{B, \alpha}$ is a co-dimension one $K$-subspace of $K[z]$ unless $\alpha=0$, in which case we have $N_{B, \alpha}=K[z]$.

Lemma 6.1. Let $\alpha \in K^{\ell}$ and $B \subseteq K^{n}$ be as above. Then $N_{B, \alpha}$ is an ideal of $K[z]$ iff the cardinal number $\left|S_{\alpha}\right| \leq 1$.

Proof: The $(\Leftarrow)$ part is obvious. To show the $(\Rightarrow)$ part, we assume $\left|S_{\alpha}\right| \geq 2$ and derive a contradiction as follows.

Without losing any generality, we assume that $\alpha_{1} \neq 0$. Since $\left|S_{\alpha}\right| \geq$ 2 , it is easy to see that there exists $f(z) \in N_{B, \alpha}$ such that $f\left(u_{1}\right) \neq 0$. Let $g(z) \in K[z]$ such that $g\left(u_{1}\right) \neq 0$ and $g\left(u_{i}\right)=0$ for all $2 \leq i \leq$ 
$n$. Then $(f g)\left(u_{1}\right) \neq 0$ and $(f g)\left(u_{i}\right)=0$ for all $2 \leq i \leq n$, whence $f g \notin N_{B, \alpha}$. But this contradicts our assumption that $N_{B, \alpha}$ is an ideal of $K[z]$.

The lemma above determines all $\alpha \in K^{\ell}$ such that $N_{B, \alpha}$ is an ideal of $K[z]$. To see for which $\alpha \in K^{\ell}, N_{B, \alpha}$ is a Mathieu subspace of $K[z]$, we have the following result proved in Proposition 4.6 in [Z4].

Proposition 6.2. Let $\alpha \in K^{\ell}$ and $B \subseteq K^{n}$ be as above. Then $N_{B, \alpha}$ is a Mathieu subspace of $K[z]$ iff $\alpha \in \Omega_{\ell}$.

Note that the proof for the proposition above in [Z4] is under the convenient condition $\alpha \in\left(K^{\times}\right)^{\ell}$. But it is easy to see that the proof actually goes through without this extra condition.

The first main result of this section is the following proposition.

Proposition 6.3. Let $\alpha \in K^{\ell}$ and $B \subseteq K^{n}$ be as above. Then we have

$$
\begin{aligned}
\sigma\left(N_{B, \alpha}\right) & =\left\{f(z) \in K[z]|| S_{\alpha_{f, B}} \mid \leq 1\right\} . \\
\tau\left(N_{B, \alpha}\right) & =\left\{f(z) \in K[z] \mid \alpha_{f, B} \in \Omega_{\ell}\right\} .
\end{aligned}
$$

Proof: Note first that for each $f(z) \in K[z]$, by Eqs. (6.1) and (6.2) it is easy to see that the following equation holds:

$$
\left(N_{B, \alpha}: f\right)=N_{B, \alpha_{f, B}} \text {. }
$$

Then with the equation above, Eq. (6.3) and (6.4) follow immediately from Lemma 6.1 and Proposition 6.2, respectively.

Remark 6.4. Let $\alpha \in K^{\ell}$ such that $\left|S_{\alpha}\right| \geq 2$. Then by Eq. (6.3), we see that the set $\sigma\left(N_{B, \alpha}\right)$ of the stable elements of the $K$-subspace $N_{B, \alpha} \subset K[z]$, as contrasted to all other examples discussed in this paper, is actually not closed under the addition of the $K[z]$-module $K[z]$.

Corollary 6.5. Let $\alpha \in K^{\ell}$ and $B \subseteq K^{n}$ be as above. Denote by $z_{\alpha}(B)$ the ideal of $K[z]$ consisting of the polynomials $f(z)$ such that $f\left(u_{i}\right)=0$ for all $i \in S_{\alpha}$. Then we have

$$
z_{\alpha}(B)=N_{B, \alpha} \cap \sigma\left(N_{B, \alpha}\right)=N_{B, \alpha} \cap \tau\left(N_{B, \alpha}\right) .
$$

Consequently, $\mathcal{Z}_{\alpha}(B)$ is the (unique) ideal of $K[z]$ that is maximum among all the ideals of $K[z]$ contained in the $K$-subspace $N_{B, \alpha}$.

Proof: First, by Eqs. (6.2) and (6.3), it is easy to see that we do have

$$
z_{\alpha}(B)=N_{B, \alpha} \cap \sigma\left(N_{B, \alpha}\right)
$$


Then the corollary follows immediately from Theorem 3.11 (by viewing $K[z]$ as the $K[z]$-module in the canonical way).

Next, we assume $K=\mathbb{C}$ and $n=1$, and consider the following family of $\mathbb{C}$-subspaces of the polynomial algebra $\mathbb{C}[z]$ in one variable $z$.

Let $a, b \in \mathbb{C}$ with $a \neq b$. For each $q(z) \in \mathbb{C}[z]$, we set

$$
N_{q}:=\left\{f \in \mathbb{C}[z] \mid \int_{a}^{b} f(z) q(z) d z=0\right\} .
$$

It is easy to see that $N_{q}$ is a co-dimension one $\mathbb{C}$-subspace of $\mathbb{C}[z]$ unless $q(z)=0$, in which case we have $N_{q}=\mathbb{C}[z]$.

Lemma 6.6. For every $0 \neq q(z) \in \mathbb{C}[z], N_{q}$ contains no nonzero ideals of $\mathbb{C}[z]$. In particular, $N_{q}$ itself is not an ideal of $\mathbb{C}[z]$.

Proof: Note first that by changing the variable $z \rightarrow(b-a) z+a$ and replacing $q(z)$ by $q((b-a) z+a)$, we may assume that $a=0$ and $b=1$.

Now, we assume otherwise. Then there exists $0 \neq h \in \mathbb{C}[z]$ such that $(h) \subseteq N_{q}$, where $(h)$ is the ideal of $\mathbb{C}[z]$ generated by $h(z)$. But, on the other hand, let $\bar{q}$ and $\bar{h}$ be the complex conjugates of $q$ and $h$, respectively. Then $(\bar{q} \bar{h})(h q)$ is continuous and has positive values at all but finitely points in the interval $[0,1]$, whence $\int_{a}^{b}(\bar{q} \bar{h})(h q) d z>$ 0 . Consequently, we have $(\bar{q} \bar{h}) h \in(h)$ but $(\bar{q} \bar{h}) h \notin N_{q}$, which is a contradiction.

The second main result of this section is the following proposition.

Proposition 6.7. For each $0 \neq q(z) \in \mathbb{C}[z]$, we have

$$
\begin{aligned}
& \sigma\left(N_{q}\right)=\{0\}, \\
& \tau\left(N_{q}\right)=N_{q}^{c} \cup\{0\} .
\end{aligned}
$$

In order to prove the proposition above, we need the following theorem proved by F. Pakovich $[\mathrm{P}]$.

Theorem 6.8. Let $a \neq b \in \mathbb{C}$ and $f(z), q(z) \in \mathbb{C}[z]$ such that for all $m \geq 1$, we have

$$
\begin{gathered}
\int_{a}^{b} q(z) d z \neq 0 \\
\int_{a}^{b} f^{m}(z) q(z) d z=0 .
\end{gathered}
$$

Then $f(z)=0$. 
Corollary 6.9. Let $0 \neq q(z) \in \mathbb{C}[z]$. Then $N_{q}$ is a Mathieu subspace of $\mathbb{C}[z]$ iff $\int_{a}^{b} q(z) d z \neq 0$.

Proof: The $(\Leftarrow)$ part follows immediately from Eq. (6.8), Theorem 6.8 and Lemma 2.1. To show the $(\Rightarrow)$ part, we assume $\int_{a}^{b} q(z) d z=0$, and derive a contradiction as follows.

Note first that by Proposition 2.6, it is easy to see that Mathieu subspaces of $\mathbb{C}[z]$ are preserved by automorphisms of the polynomial algebra of $\mathbb{C}[z]$. So, by applying the change of the variables in the proof of Lemma 6.6, we may assume that $a=0$ and $b=1$.

Since $\int_{0}^{1} q(z) d z=0$, we have $1 \in N_{q}$. Since $N_{q}$ by assumption is a Mathieu subspace of $\mathbb{C}[z]$, by Lemma 2.3 we have $N_{q}=\mathbb{C}[z]$. In particular, the complex conjugate $\bar{q}$ of $q$ lies in $N_{q}$, i.e., $\int_{0}^{1} \bar{q} q d z=0$. But, this is impossible, as argued in the proof of Lemma 6.6. Therefore, we get a contradiction.

Proof of Proposition 6.7: For each $h(z) \in \mathbb{C}[z]$, by Eq. (6.8) it is easy to see that we have

$$
\left(N_{q}: h\right)=N_{h q}
$$

Hence by Lemma 6.6 and the equation above, we get Eq. (6.9).

To show Eq. (6.10), note first that by Lemma 6.6 and Theorem 3.11. we have $\tau\left(N_{q}\right) \subseteq N_{q}^{c} \cup\{0\}$.

Now, let $h(z) \in N_{q}^{c}$. Then we have $\int_{a}^{b} h(z) q(z) d z \neq 0$. Applying Corollary 6.9 to the polynomial $h q$, we see that $N_{h q}$ is a Mathieu subspace of $\mathbb{C}[z]$. Then by Eq. (6.13) above, we get $h \in \tau\left(N_{q}\right)$. Hence, we have $N_{q}^{c} \subseteq \tau\left(N_{q}\right)$. Moreover, by Lemma 3.3, $\left.i i\right)$ we also have $0 \in \tau\left(N_{q}\right)$, whence Eq. (6.10) follows.

Acknowledgments The author would like to thank the referee and Michiel de Bondt for pointing out some mistakes and misprints in the earlier version of this paper.

\section{REFERENCES}

[AE] Adjamagbo P. K., van den Essen A., A Proof of the Equivalence of the Dixmier, Jacobian and Poisson Conjectures. Acta Math. Vietnam. 32 (2007), no. 2-3, 205-214. [MR2368008].

[BCW] Bass H., Connell E., Wright D., The Jacobian Conjecture, Reduction of Degree and Formal Expansion of the Inverse. Bull. Amer. Math. Soc. 7, (1982), 287-330. [MR 83k:14028].

[BK] Belov-Kanel A., Kontsevich M., The Jacobian Conjecture Is Stably Equivalent to the Dixmier Conjecture. (English, Russian summary) Mosc. Math. J. 7 (2007), no. 2, 209-218, 349. [MR2337879]. 
[D] Dixmier J., Sur les algèbres de Weyl. Bull. Soc. Math. France, 96 (1968), 209-242. [MR0242897].

[E1] van den Essen A., Polynomial Automorphisms and the Jacobian Conjecture. Progress in Mathematics, 190. Birkhäuser Verlag, Basel, 2000. [MR1790619].

[E2] van den Essen A., The Amazing Image Conjecture. Preprint. See arXiv:1006.5801v1 [math.AG].

[EWiZ] A. van den Essen, R. Willems and W. Zhao, Some Results on the Vanishing Conjecture of Differential Operators with Constant Coefficients. Under submission. See also arXiv:0903.1478 [math.AC].

[EWrZ1] A. van den Essen, D. Wright and W. Zhao, Images of Locally Finite Derivations of Polynomial Algebras in Two Variables. Under submission. See also arXiv:1004.0521v1 [math.AC].

[EWrZ2] A. van den Essen, D. Wright and W. Zhao, On the Image Conjecture. Preprint. See arXiv:1008.3962 [math.RA].

[EZ] van den Essen A., Zhao W., Mathieu Subspaces of Univariate Polynomial Algebras. In preparation.

[FPYZ] Francoise J. P., Pakovich F., Yomdin Y., Zhao W., Moment Vanishing Problem and Positivity: Some Examples. To appear in Bulletin des Sciences Mathématiques. doi:10.1016/j.bulsci.2010.06.002.

[K] Keller O. H., Ganze Gremona-Transformationen. Monats. Math. Physik 47 (1939), no. 1, 299-306. [MR1550818].

[Ma] Mathieu O., Some Conjectures about Invariant Theory and Their Applications. Algèbre non commutative, groupes quantiques et invariants (Reims, 1995), 263-279, Sémin. Congr., 2, Soc. Math. France, Paris, 1997. [MR1601155].

[P] Pakovich F., On Rational Functions Orthogonal to All Powers of a Given Rational Function on a Curve. Preprint. See arXiv:0910.2105 [math.CV].

[T] Tsuchimoto Y., Endomorphisms of Weyl Algebra and p-Curvatures. Osaka J. Math. 42 (2005), no. 2, 435-452. [MR2147727].

[WZ] Willems R., Zhao W., Analogue of the Duistermaat-van der Kallen Theorem for Group Algebras. In preparation.

[Z1] Zhao W., Hessian Nilpotent Polynomials and the Jacobian Conjecture, Trans. Amer. Math. Soc. 359 (2007), no. 1, 249-274 (electronic). [MR2247890]. See also math.CV/0409534.

[Z2] Zhao W., A Vanishing Conjecture on Differential Operators with Constant Coefficients, Acta Mathematica Vietnamica, vol 32 (2007), no. 3, 259-286. [MR2368014]. See also arXiv:0704.1691v2 [math.CV].

[Z3] Zhao W., Images of Commuting Differential Operators of Order One with Constant Leading Coefficients. J. Alg. 324 (2010), no. 2, 231-247. See also arXiv:0902.0210 [math.CV].

[Z4] Zhao W., Generalizations of the Image Conjecture and the Mathieu Conjecture. J. Pure Appl. Algebra. 214 (2010), no. 7, 1200-1216. [MR2586998]. See also arXiv:0902.0212 [math.CV].

[Z5] Zhao W., New Proofs for the Abhyankar-Gurjar Inversion Formula and the Equivalence of the Jacobian Conjecture and the Vanishing Conjecture. To appear in Proc. AMS. See also arXiv:0907.3991 [math.AG]. 
[Z6] Zhao W., Mathieu Subspaces of Associative Algebras. Under submission. See also arXiv:1005.4260 [math.RT].

Department of Mathematics, Illinois State University, Normal, IL 61790-4520. E-mail: WZHAO@ILSTU.EDU. 